
A. de la Maza
L. Coderch
O. Lopez
J. Baucells
J.L. Parra

\section{Permeability changes in liposomes modeling the stratum corneum lipid composition caused by surfactants}

Dr. A. de la Maza (ख) . L. Coderch

O. Lopez J.L. Parra

Departamento de Tensioactivos

C.I.D.-C.S.I.C.

Calle Jorge Girona 18-26

08034 Barcelona, Spain

\section{J. Baucells}

Universidad Autonoma de Barcelona

(U.A.B.)

Facultad de Veterinaria

Bellaterra

08193 Barcelona, Spain

\begin{abstract}
The alterations caused by the surfactants sodium dedecyl sulfate (SDS); sodium dodecly ether sulfate (SDES); Triton X-100 (OP.10EO) and dodecyl betaine (D-Bet) in the permeability of liposomes formed by a lipid mixture modeling the stratum corneum composition (40\% ceramides, $25 \%$ cholesterol, $25 \%$ plamitic acid and $10 \%$ cholesteryl sulfate) were investigated. The SC liposomes/surfactant sublytic interactions were mainly ruled by the action of surfactant monomers in all cases. The OP-10EO showed the highest ability to alter the permeability of bilayers and the highest affinitywith these structures, whereas D-Bet showed the lowest tendencies. Although SDS and SDES exhibited similar activity at $50 \% \mathrm{CF}$ release (similar Re values), the SDES
\end{abstract}

appeared to be more active at $100 \%$ $\mathrm{CF}$ release, its affinity with bilayers being also increased. Different trends in the evolution of Re and $K$ were observed when comparing the results obtained with those reported for phosphatidylcholine (PC) liposomes. Thus, wbereas SC liposomes were more resistant to the action of surfactants, the surfactant affinity with $\mathrm{SC}$ bilayers was always greater than that reported for PC ones.

Key words Stratum corneum liposomes - stratum corneum liposomes/surfactant inferactions permeability alteration carboxyfluorescein release surfactant/stratum corneum lipids molar ralios - surfactant partition coefficients

\section{Introduction}

The stratum corneum (SC), the outermost layer of mammalian epidermis, consists of fat cells (corneocytes) that are separated by an intercellular matrix mainly composed of lipids. These lipids are organized into bilayers that have been postulated both to account for the permeability properties of SC and possibly to ensure the cohesiveness between corneocytes $[1,2]$.

In order to find our whether SC lipids could form bilayers, Wertz and Abraham 13-51 prepared liposomes from lipid mixtures approximating the composition of SC lipids at physiological $\mathrm{pH}$. These authors also investigated the interaction of these liposomes with the anionic surfactant sodium dodecyl sulfate (SDS) to study the deleterious effect of this surfactant on human skin [6]. Furthermore, Blume et al. reported that mixing PC liposomes with lipid model mixtures for SC lipids could be one mechanism contributing to the enhancement of the permeability of the skin to lipid vesicles [7].

In recent papers, we have studied the interactions of different surfactants and surfactant mixtures with simplified membrane models such as PC liposomes [8-10] as well as the formation and characterization of liposomes formed with mixtures of four commercially available 
synthetic lipids approximating the composition of stratum corneum [11]. In the present work, we seek to extend these investigations by characterizing at subsolubilizing level the surfactant to lipid molar ratios and the partition coefficients of different surfactants between these bilayers and the aqueous medium. The results obtained in this study provide uew information about the effect caused by these surfactants on the permeability of SC bilayers taking into account the different physico-chemiçal characteristics of the amphiphilic compounds tested.

\section{Materials and methods}

Sodium dodecyl sulfate (SDS) was obtaiiied from Merck (Darmstadt, Germany) and further purified by a column chrornatographic method [12]. Sodium dodecyl ether sulfate (SDES) was supplied by Tenneco S.A. (Barcelona Spain). The latter was a commercial-grade product with an active matter of $28.8 \%, 2.5$ average in ethylene oxide units and the following average in alkyl chain: $\mathrm{C}-10,3.9 \%, \mathrm{C}-12$, $68.1 \% ; \mathrm{C}-14,22.2 \%$ and $\mathrm{C}-16,4.9 \%$. Nonionic surfactant Triton X-100 (OP-10EO), octylphenol ethoxylated with 10 units of ethylene oxide and an active matter of $100 \%$ was purchased from Rohm and Haas (Lyon, France). The amphoteric surfactant $\mathrm{N}$-dodecyl-N,N-dimethylbetaine (D-Bet) was specially prepared by Albright and Wilson, Ltd. (Warley, West Midlands, U.K.); the active matter was $30 \%$ in aqueous solution and the amiuo free contents was 0.20\%. Piperazine-1,4-bis(2-ethanesulphonic acid) (PIPES buffer) obtained from Merck (Darmstadt, Germany) was prepared as $20 \mathrm{mM}$ PIPES buffer adjusted to $\mathrm{pH} 7.20$ with $\mathrm{NaOH}$, containing $110 \mathrm{mM} \mathrm{Na}_{2} \mathrm{SO}_{4}$. The starting material 5(6)-carboxyfluorescein (CF), was obtained from Eastman Kodak (Rochester, NY) and further purified by a column chromatographic method [13]. Reagent-grade organic solvents, ceramides type III (Cer) and cholesterol (Chol) were supplied by Sigma Chemical Co. (St Louis, $\mathrm{MO}$ ) and palmitic acid (PA) (reagent grade) was purchased from Merck. Cholesteryl sulfate (Chol-sulf) was prepared by reaction of cholesterol with excess chlorosulphonic acid in pyridine and purified chromatographically. The molecular weight of ceremides type III was determined by low-resolution fast atom bombardment mass spectrometry (FAB-MS) using a Fisons VG Auto Spec Q (Manchester, U.K.) with a caesium gun operating at $20 \mathrm{Kv}$. The lipids of the bighest purity grade available were stored in chloroform/methanol $2: 1$ under nitrogen at $-20^{\circ} \mathrm{C}$ until use.

Liposomes formed by a mixture of lipids modeling the composition of the SC (40\% Cer, $25 \%$ Chol, $25 \%$ PA and $10 \%$ Chol-sulf) were prepared following the method described by Wertz et al. [3]. Vesicles of defined size (about
$200 \mathrm{~nm}$ ) were obtained by extrusion techniques (VETs). Vesicles were freed of unencapsulated fluorescent dye by passage through Sephadex G-50 medium resin (Pharmacia, Uppsala, Sweden) by column chromatography to study the bilayer permeability changes due to the presence of different surfactants.

The bilayer lipid composition after liposome preparation was determined using thin-layer chromatography (TLC) coupled to an automated flame ionization detection (FID) system (latroscau MK-5, Iatron Lab. Inc. Tokyo, Japan) [14].

In order to find out whether all the mixture lipid components formed liposomes, vesicular dispersions were analyzed for these lipids [14]. The dispersions were then spun at $140000 \mathrm{~g}$ at $37^{\circ} \mathrm{C}$ for $4 \mathrm{~h}$ to remove the vesicles [15]. The supernatants were tested again for these componenis. No lipids were detected in any of the supernatants.

Analyses of proton magnetic resonance ( $\left.{ }^{1} \mathrm{H} \mathrm{NMR}\right)$ were carried out at temperature ranging from $25^{\circ} \mathrm{C}$ and $90^{\circ} \mathrm{C}$ to determine the phase-transition temperature of the lipid mixture formiiig liposomes. The ${ }^{1} \mathrm{H} N \mathrm{NMR}$ spectra were recorded on a Varian Unity of $300 \mathrm{MHz}$ (Palo Alto, California, U.S.A.). The NMR spectra were measured at intervals of $5^{\circ} \mathrm{C}$. The different line widths were plotted versus the temperature, and infection point of the curve was taken as a phase-transition temperature, which showed a value of $55-56^{\circ} \mathrm{C}$.

The vesicle size distribution and the polydispersity index (PI) of liposomes after preparation was determined with dynamic light-scattering measiirements using a photon correlator spectrometer (Malvern Autosizer $4700 \mathrm{c}$ PS/MV). The studies were made by particle numher measurement at $37^{\circ} \mathrm{C}$ and using a reading angle of $90^{\circ}$. After preparaiion vesicle size distribution varied very little (lipid concentration from 0.5 to $5.0 \mathrm{mM}$ ) showing in all cases a similar value of about $200 \mathrm{~nm}$ (PI lower than 0.1), thereby indicating tbat the size distribution was very homogeneous. The size of vesicles after the addition of equal volumes of PIPES buffer and equilibration for $60 \mathrm{~min}$ showed in all cases values similar to those obtained after preparation, with a slight increase in the PI (between 0.10 and 0.12 ). Hence, the liposome preparations appeared to be reasonably stable in the absence of surfactant under the experimental conditions used in permeability studies.

The surface tensions of buffered solutions containing increasing concentrations of surfactants were measured by the ring method [16] using a Krüss tensiometer. The surfactant critical micelle concentration $(\mathrm{cmc})$ was deter mined from the abrupt change in the slope of the surface tension values versus surfactant concentration. The values obtained for each surfactant tested are given in Table 1.

In the analysis of the equilibrium partition model proposed by Schurtenberger [17] for bile salt/lecithin systems, 
Table 1 Surfactant to lipid molar ratios (Re), partition coefficients $(K)$ and surfactant concentrations in the aqueous medium $\left(S_{\mathrm{w}}\right)$ resulting in the subsolubilizing interaction ( $50 \%$ and $100 \%$ of CF release) of OP-10EO, SDS, SDES and D-Bet surfactants with SC liposomes. The regression coefficients of the straight lines obtained are also included

\begin{tabular}{llllllllll}
\hline & $\begin{array}{l}\text { CMC } \\
(\mathrm{mM})\end{array}$ & $\begin{array}{l}S_{\mathrm{W}, 50 \% \mathrm{CF}} \\
(\mathrm{mM})\end{array}$ & $\begin{array}{l}S_{\mathrm{W} .100 \% \mathrm{FC}} \\
(\mathrm{mM})\end{array}$ & $\begin{array}{l}\mathrm{Re}_{50 \% \mathrm{CF}} \\
\text { mole/mole }\end{array}$ & $\begin{array}{l}\mathrm{Re}_{100 \% \mathrm{CF}} \\
\text { mole/mole }\end{array}$ & $\begin{array}{l}K_{50 \% \mathrm{CF}} \\
\left(\mathrm{mM}^{-1}\right)\end{array}$ & $\begin{array}{l}K_{100 \% \mathrm{CF}} \\
\left(\mathrm{mM}^{-1}\right)\end{array}$ & $\begin{array}{l}r^{2} \\
(50 \% \mathrm{CF})\end{array}$ & $\begin{array}{l}r^{2} \\
(100 \% \mathrm{CF})\end{array}$ \\
\hline OP-10EO & 0.15 & 0.039 & 0.089 & 0.190 & 0.448 & 4.09 & 3.47 & 0.993 & 0.994 \\
SDS & 0.50 & 0.083 & 0.289 & 0.350 & 1.0 & 3.12 & 1.70 & 0.994 & 0.996 \\
SDES & 0.12 & 0.086 & 0.105 & 0.351 & 0.733 & 3.02 & 3.99 & 0.995 & 0.993 \\
D.Bet & 1.25 & 0.418 & 0.838 & 0.653 & 0.756 & 0.95 & 0.51 & 0.997 & 0.995 \\
\hline
\end{tabular}

Lichtenberg [18] and Almog et al. [15] have shown that for a mixing of lipids (at a lipid concentration $\mathrm{L}(\mathrm{mM})$ ) and siirfactant (at a concentration $S_{\mathrm{T}}(\mathrm{mM})$ ), in dilute aqueous media, the distribution of surfactant between lipid bilayers and aqueous media obeys a partition coefficient $\mathrm{K}$, given (in $\mathrm{mM}^{-1}$ ) by

$$
\mathrm{K}=\operatorname{Re} / S_{\mathrm{W}}[1+\mathrm{Re}] \text {, }
$$

where $\mathrm{Re}$ is the effective molar ratio of surfactant to lipid in the bilayers $\left(\operatorname{Re}=S_{\mathrm{B}} / L, S_{\mathrm{B}}\right.$ being the surfactant concentration in bilayers) and $S_{\mathrm{W}}$ is the surfactant concentration in the aqueous medium (mM). This approach is consistent with the experimental data offered by Lichtenberg [18] and Almog [15] for different surfactant lipid mixtures over wide ranges of Re values. Given that the range of lipid concentrations used in the mixture is similar to that used by Almog to test his equilibrium partition model, the $\mathrm{K}$ parameter has been determined using this equation.

The determination of these parameters can be carried out on the basis of the linear dependence existing between the surfactant concentrations required to achieve 50 and $100 \%$ of CF release and the SC lipid concentration (SCL), which can be described by the equations

$S_{\mathrm{T}}=S_{\mathrm{W}, 50 \% \mathrm{CF}}+\mathrm{Re}_{50 \% \mathrm{CF}}[\mathrm{SCL}]$,

$S_{\mathrm{T}^{\prime}}=S_{\mathrm{W}, 100 \% \mathrm{CF}}+\operatorname{Re}_{100 \% \mathrm{CF}}[\mathrm{SCL}]$,

where the $\operatorname{Re}_{50 \% \mathrm{CF}}, \operatorname{Re}_{100 \% \mathrm{CF}}$ and the aqueous concentration of surfactant $S_{\mathrm{W}, 50 \% \mathrm{CF}}$ and $S_{\mathrm{W}, 100 \% \mathrm{CF}}$ are in each curve, respectively, the slope and the ordinate at the origin (zero lipid concentration).

The permeability changes caused by the presence of different surfactant in SC liposomes were determined qiiantitatively by monitoring the increase in the fluorescence intensity of the liposome suspensions due to the $\mathrm{CF}$ released from the interior of vesicles to the bulk aqueous phase [10]. Fluorescence measurements were made with a spectrofluorophotometer Shimadzu RF-540 equipped with a thermoregulated cell compartment (Kyoto Japan). On excitation at $495 \mathrm{~nm}$, a fluorescence maximum emission of $\mathrm{CF}$ was obtained at $515.4 \mathrm{~nm}$.

\section{Results and discussion}

In preliminary experiments, we determined the suitable sonication temperature of the lipid mixture investigated by preparing liposomes at temperatures approximating its phase-transition temperatiire $\left(55-56^{\circ} \mathrm{C}\right)$. II was found that temperatures exceeding this temperature by more than $10^{\circ} \mathrm{C}$ caused clear alterations in Cer and Chol-sulph. As a consequence, lipid mixture was sonicated at 60 "C. To determine the time needed to obtain a constant level of $\mathrm{CF}$ release of liposomes in the lipid concentration range investigated, a kinetic study of the interaction of various surfactants with SC liposomes was carried out. Liposomes were treated with a constant subsolubilizing surfactant concentration $(0.5 \mathrm{mM})$ and subsequent changes in permeability were studied as a fuuction of time. The results obtained for $1.0 \mathrm{mM}$ lipid concentration are given in Fig. 1. About 60 min was needed to achieve a constant level of $\mathrm{CF}$ release. Hence, changes in permeability were studied $60 \mathrm{~min}$ after addition of surfactants to the liposomes at $37^{\circ} \mathrm{C}$. This finding contracts with that reported for the interaction of these surfactants with PC liposomes, where the time needed to obtain a constant level of $\mathrm{CF}$ release was always clearly lower [19]. The CF release of SC liposomes in the absence of surfactant in this period of time was negligible.

To determine the Re and $S_{\mathrm{W}}$ parameters at two stublytic levels (50 and $100 \% \mathrm{CF}$ release), a systematic investigation of permeability changes caused by the addition of difîerent surfactants was carried out for various SC lipid concentrations (from 0.5 to $5.0 \mathrm{mM}$ ). The curves obtained for the anionic surfactant SDS are given in Fig. 2. The surfactant concentrations resulting in 50 and $100 \%$ of $\mathrm{CF}$ release for each surfactant tested were graphically obtained and plotted versus lipid concentration. An acceptable linear relationship was established in each case. These results are plotted in Figs. 3A (50\% CF release) and B (100\% CF release) respectively. The straight lines obtained corresponded to the aforementioned Eqs. (2) and (3) from which Re and $\mathrm{S}$, were determined. These parameter 


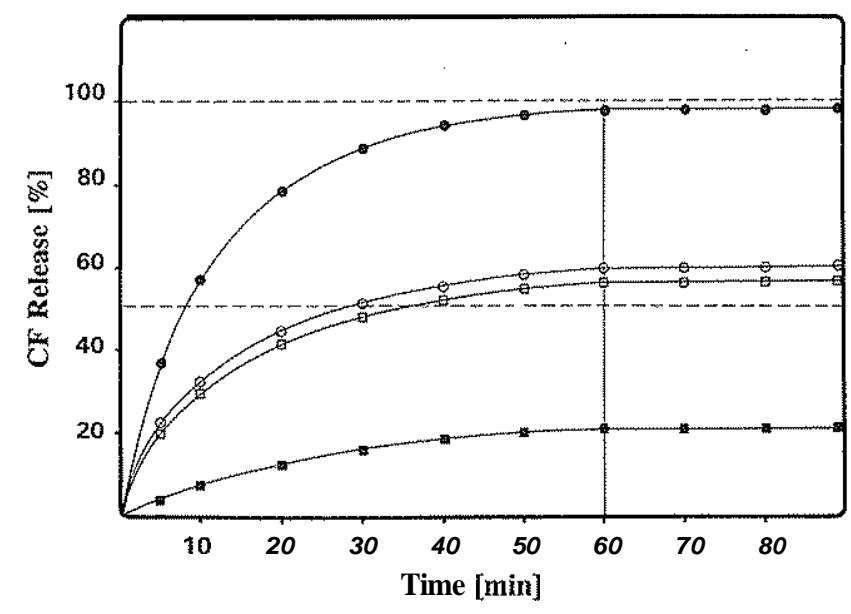

Fig. 1 Time curves of the release of CF trapped into SC liposomes caused by the addition of a constant concentration $(0.5 \mathrm{mM})$ of OP-IOEO (•), SDS (D), SDES (O) and D-Bet (a). The lipid concentration was $5.0 \mathrm{mM}$

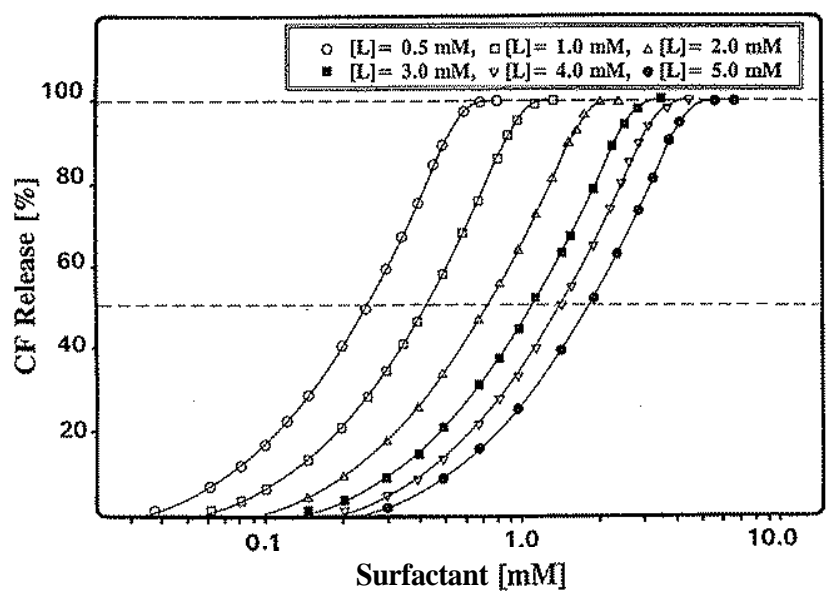

Fig. 2 Percentage changes in $\mathrm{CF}$ release of SC liposomes, (lipid concentration ranging from 0.5 to $5.0 \mathrm{mM}$ ), induced by the prescnce of increasing concentrations of SDS. Lipid concentrations: $0.5 \mathrm{mM}$ $(0), 1.0 \mathrm{mM}(\square), 2.0 \mathrm{mM}(\Delta), 3.0 \mathrm{mM}(\mathbf{\Delta}), 4.0 \mathrm{mM}(\nabla), 5.0 \mathrm{mM}(\bullet)$

including the regression coefficients $\left(\mathrm{r}^{2}\right)$ of the straight lines are also given in Table 1.

The $S_{\mathrm{W}}$ values increased as the CF release percentage rose, although showing smaller values than those corresponding surfactant cmc's in all cases. This finding suggests that the surfactant-liposome interaction must be ruled mainly by the action of surfactant monomers, unlike the behavior of the surfactants of solubilization of phospholipid bilayers, where micelle formation plays a very important role [8]. These findings are in agreement with those reported for subsolubilizing and solubilizing interactions of these surfactants with PC unilamellar liposomes in the same buffered working medium $[19,20]$
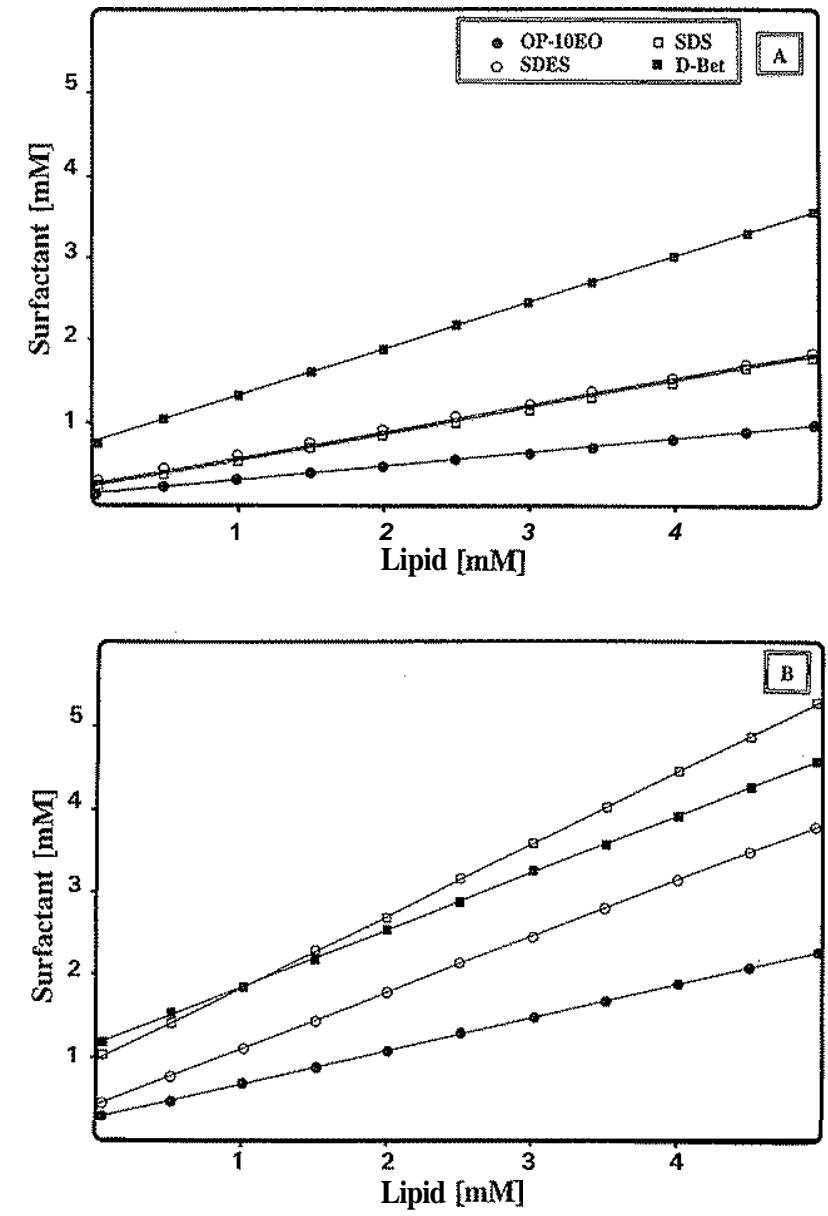

Fig. 3 Surfactant concentrations resulting in A) $50 \%$ of CF release and B) $100 \%$ of $\mathrm{CF}$ release versus lipid concentration of liposome

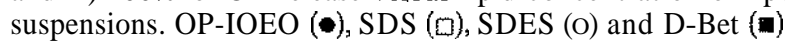

As for the Re parameter, this value increased as the $\mathrm{CF}$ release percentage rose, regardless of the chemical structure of the surfactant tested. Bearing in mind that the surfactant capacity to alter the permeability of bilayers is inversely related to the Re parameter, the maximum activity at $50 \%$ of $\mathrm{CF}$ release corresponded to the nonionic surfactant OP-IOEO (lowest Re values) and the minimum to the amphoteric D-Bet (highest Re values), the anionics SDS and SDES exhibiting intermediate values. Similar behavior was detected at $100 \%$ of CF release except for the SDES, which exhibited higher bilayer activity than the SDS. This effect may be attributed to the presence of 2.5 EO units in the SDES molecular structure, given that this is the unique structural difference between both anionic surfactants.

Comparison of the Re values with those reported for the interaction of these surfactants with PC unilamellar liposomes [19] reveals that ability of these surfactants to alter the permeahility of SC bilayers (50\% CF release) 
appeared to be lesser (higher Re values) than that reported for PC unilamellar liposomes in all cases. Thus, SC bilayer structures appeared to be more resistant to the surfactant perturbations at the sublytic level investigated.

The surfactant partition coefficients between SC bilayers and aqueous medium both at 50 and $100 \%$ of $\mathrm{CF}$ release indicate that the OP-10EO molecules had the highest affinity with bilayers (maximum $\mathrm{K}$ values), whereas the amphoteric surfactant D-Bet showed the lowest (minimum $\mathrm{K}$ values). As for the nonionic surfactants, although at $50 \%$ of CF release SDS showed higher affinity with bilayers than SDES, at $100 \% \mathrm{CF}$ release the affinity of these surfactants with SC liposomes exhibited opposite tendencies. As discussed above, this effect may be attrihuted to the presence of $2.5 \mathrm{EO}$ units in the SDES molecular structure.

The fact that OP-IOEO, SDS and D-Bet showed at $100 \%$ C F release lower $K$ values than those for $50 \%$ could be explained assuming that the low Re (approx in the interval of C F release between 30 and $60 \%$ ) only the outer vesicle leaflet was available for interaction with surfactant molecules, the bindiiig of additional molecules to bilayers being hampered at slightly higher Re values. These findings are in agreement with those reported by Schubert et al. for sodium cholate [21] and with our previous invesigations involving the overall interaction of OP-10EO and SDS with PC liposomes $[8,10]$. This behavior contrasts with the increased bilayer affinity exhibited by SDES at $100 \%$ CF release. This effect may be attributed to the specific structure of this anionic surfactant, which could affect the rate of flip-fiop of surfactant molecules (or permeabilization of the bilayers to surfactants), thus making the inner monolayer available for the interaction of added surfactant. Comparison of the $\mathrm{K}$ values obtained with those reported for the interaction of these surfactants with PCliposomes indicates that the surfactant affinity with SC bilayers appeared to be greater than that for PC ones in al! cases $[8,10,19]$.

In general terms, different trends in the interaction of these surfactant with SC and PC liposomes may be observed at subsolubilizing level. Thus, whereas SC liposomes appeared to be more resistant to the action of surfactant monomers the affinity of these compoiinds with SC structures appeared to be greater than with PC ones. Thus, although a greater number of surfactant molecules was needed to produce alternations in SC bilayers, these moleciiles showed increased affinity with these structures. This behavior is directly correlated with the lesser free surfactant concentrations ( $S_{\mathrm{W}, 50 \% \mathrm{CF}}$ and $\left.S_{\mathrm{W}, 100 \% \mathrm{CF}}\right)$ obtained in the interaction of these surfactants with SC liposomes $[8,10,19]$. We are aware of the fact that the lipids used in this work are not exactly the same as'those existing in the stratum corneum. Nevertheless, our approach may be useful for stiidying the interaction of different surfactants with these lipid structures and comparing the Re ratios for different sublytic interaction steps with those obtained for PC liposomes. This comparison could be also useful in establishing a criterion for the evaluation of the activity of these surfactants in human skin.

Acknowledgments This work was supported by funds from DGICYT (Dirección General de Investigación Cientifica y Técnica) (Prog. no. PB94.0043), Spain. We are grateful to Mr. G. von Knorring for expert technical assistance.

\section{Refetences}

1. Friberg SE, Goldsmith LB, Kayali I, Suhaimi H (1991) In: Bender M (ed) Interfacial Phenomena in Biological Systems, Surfactant Science Series, Vol. 39, Chap. 1. Marcel Dekker, Inc., New York

2. Bouwstra JA, Gooris GS, Bras W, Downing DT (1995) J Lipid Res 36685-695

3. Wertz PW. Abraham W, Landman L, Downing DT (1986) I Invest Dermatol $87.582-584$

4. Wertz PW (1992) In Braun-Falco O, Korting HC, Maibach H (eds) Liposome Dermatics (Griesbach Conference). Springer, Berlin, pp 38-43.

5. Abraham W. Wertz PW, Landman L, Downing DT (1987) J Invest Dermatol $88: 212-214$

6. Downing DT. Abraham W. Weoner BK. Willman KW. Marshall JM (1993) Arch Dermatol Res 285 151-157
7. Blume A, Jansen M, Ghyczy M, Gareișs J (1993) Int J Pharm 99:219-228

8. de la Maza A, Parra JL (1994) Biochem J 303:907-914

9. dc la Maza A, Parra JL (1994) Eur J Biochem 2261029--1038

10. de la Maza A. Parra JL (1995) J Am Oll Chem Soc 72:131-136

11. de la Maza A, Manich AM, Coderch L. Bosch P, Parra JL (1995) Colloids Sufaces A: Physicochem Eng Aspects $101: 9 \div 19$

12. Rosen, MJ (1989) J Colloid Interface Sci 79:587-588

13. Weistein JN, Ralston E, Leserman LD, Klausner RD, Dragsten P. Henknrt P. Blumenthal R (1986) In Geogoriadis G (ed) Liposome Tcchnology, Vol Ill, Chap 13. CRC Press, Boca Raton, FL
14. Ackman RG, McLeod CA, Banergee AK (1990) J Planai Chrom 3:450 490

15. Almog S, Litman BJ, Wimley W, Cohen $\mathrm{J}$, Wachtel EJ, Barenholz Y, Ben-Shaul A, Lichtenberg D (1990) Biochemistry 294582-4592

16. Lunkenheimer K, Wantke D (1981)Colloid Polymer Sci. 259:354-366

17. Schurtenberger $P$, Mazer N, Känzig W (1985) J Phys Chem 89:1042 - 1049

18. Lichtenberg D (1985) Biochim Biophys Acta $821: 470-478$

19. de la Maza A, Sanchez J, Parra JL, Garcia MT, Ribosa I (1991) J Am Oil Chem Soc 68:315 319

20. de la Maza A. Parra JL (1993) J Am Oil Chem Soc 70:699-706

21. Schubert R, Beyer K, Wolburg $H$, Schmidt KH (1986) Biochemistry $25: 5263-5269$ 